\title{
Coupling of carbon nanotubes to metallic contacts
}

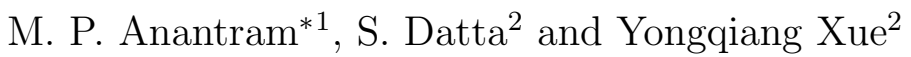 \\ ${ }^{1}$ NASA Ames Research Center, Mail Stop T27A-1, Moffett Field, CA, USA 94035-1000 \\ 2 Department of Electrical and Computer Engineering, Purdue University, West Lafayette, IN
}

$47907-1285$

\begin{abstract}
The modeling of carbon nanotube-metal contacts is important from both basic and applied view points. For many applications, it is important to design contacts such that the transmission is dictated by intrinsic properties of the nanotube rather than by details of the contact. In this paper, we calculate the electron transmission probability from a nanotube to a free electron metal, which is side-contacted. If the metal-nanotube interface is sufficiently ordered, we find that k-vector conservation plays an important role in determining the coupling, with the physics depending on the area of contact, tube diameter and chirality. The main results of this paper are: (i) conductance scales with contact length, a phenomena that has been observed in experiments and (ii) in the case of uniform coupling between metal and nanotube, the threshold value of the metal Fermi wave vector (below which coupling is insignificant) depends on chirality. Disorder and small phase coherence length relax the need for k-vector conservation, thereby making the coupling stronger.
\end{abstract}

Physical Review B, vol. 61, p. 14219 (2000), APS March 1999 Meeting

Typeset using REVTEX 


\section{INTRODUCTION}

Carbon nanotubes represent an intriguing new material that has attracted much attention both from theorists and experimentalists since the early 1990s.1 Particularly exciting is the possibility of one dimensional metallic conductors at room temperature that can be used as a probe in scanning probe microscopy or as a low resistance ballistic interconnect for electron devices. physics of conduction by studying the conductance of such a one dimensional conductor at low temperatures. To exploit these possibilities it is important to understand the physics of the nanotube-metal contacts and to experimentally demonstrate low resistance contacts in a reproducible manner. The contact between carbon nanotubes and metal can occur at the end of the tube (end-contact) 5 .6 and along the circumference of the tube (side-contact) 䀢. The low contact resistance demonstrated by de Pablo et. al a strong interaction between metal and carbon atoms at the end of the nanotube, or/and due to lack of translational symmetry. 1 In comparison, the interaction between metal and carbon atoms in side-contacted nanotubes is weak.

An interesting manifestation of weak distributed coupling is that the contact resistance is inversely proportional to contact length as observed experimentally by references 2 and 8. Recently, Tersoff in a perceptive paper qualitatively discussed the importance of kvector conservation when the coupling between nanotube and metal is weak. The important physical quantities are diameter and chirality of the nanotube, Fermi wave vector of the metal, area of contact, and details of the metal-nanotube contact. In this paper, we study the physics of side-contacted nanotube-metal contacts2 2 目 by addressing how these physical quantities affect the transmission of electrons from the nanotube to the metal contact. For small diameter nanotubes, our conclusions do not fully agree with Ref. 9. We find that for small diameter armchair tubes, the threshold value of Fermi wave vector below which the conductance is very small is $2 \pi / 3 a_{0}$ and not $4 \pi / 3 a_{0}$, which is the value for graphene. $a_{0}=2.46 \AA$ is the lattice vector length of graphene. In contrast to armchair tubes, the 
threshold for zigzag tubes is zero. Our calculations also show that the conductance scales with contact length, a phenomena that has been observed experimentally in the work of Tans et. alla and Frank et. al.8

In the remainder of the introduction, we discuss the salient results using simple arguments. The method is discussed in section 1 l] and the numerical results and discussion are presented in section [II. We present our conclusions in section IV.

The first Brillouin zone of graphene touches the Fermi surface at six points (Fig. 1). Of these only two points are inequivalent (that is, do not differ by a reciprocal lattice vector). The conduction properties of graphite at low bias are controlled by the nature of eigenstates around these points. Consider a metal making uniform contact to graphene. The in-plane wave vector should be conserved when an electron tunnels from the metal to the nanotube. As a result, for good coupling between metal and graphene, the metal Fermi wave vector should be comparable to $4 \pi / 3 a_{0}$, which corresponds to the Fermi wave vector of graphene.

To discuss the case of nanotubes making contact to metal, we consider the scattering rate $\left(1 / \tau_{c-m}\right)$ from the metal to nanotube within the Born approximation,

$$
1 / \tau_{c-m} \propto<\Psi_{c}\left|H_{c-m}\right| \Psi_{m}>
$$

where, $\Psi_{m}\left(\Psi_{c}\right)$ is the metal (nanotube) wave function and $H_{c-m}$ represents the nanotubemetal coupling. The wave function of an $(\mathrm{n}, \mathrm{m})$ nanotube is $\Psi_{c} \sim e^{i k_{t} p u} \phi_{c}$, where $k_{t}$ is the axial wave vector, $u$ is the $1 \mathrm{D}$ unit cell length, $p$ is an integer representing the various unit cells and $\phi_{c}$ is a vector representing the wave function of all atoms in a unit cell. It is assumed that the wave function of the metal is separable in the axial and radial directions of the nanotube, $\left|\Psi_{m}>\sim e^{i k_{m} p u}\right| \phi_{m}>$, where $k_{m}$ is the metal wave vector component along the nanotube axis. When the coupling between the nanotube and metal is uniform, the scattering rate is [Eq. (1)],

$$
1 / \tau_{c-m} \propto t_{c-m}<\phi_{c} \mid \phi_{m}>\sum_{p} e^{i\left(k_{m}-k_{t}\right) p u},
$$

where, the summation is performed over all unit cells making contact to metal and $t_{c-m}$ represents a uniform coupling constant between the metal and nanotube. It is clear from 
Eq. (2) that provided the metal and nanotube make contact over several unit cells, wave vector conservation along the axial direction is enforced as $\sum_{p} e^{i\left(k_{m}-k_{t}\right) p u} \sim \frac{1}{u} \delta\left(k_{m}-k_{t}\right)$. The axial wave vector corresponding to $E=0$ are $2 \pi / 3 a_{0}$ and 0 for armchair and zigzag tubes respectively, and the wave vector for other chiralities varies between these two limits. As a result, the threshold value of Fermi wave vector below which coupling between an armchair (zigzag) nanotube and metal is poor is $2 \pi / 3 a_{0}(0)$. The threshold value of the metal Fermi wave vector for chiral tubes is in between that of zigzag and armchair tubes. As the diameter of the nanotube increases, wave vector conservation along the circumference becomes increasingly important, as the strip approaches a graphene sheet.

\section{METHOD}

The method used to calculate transmission probability is essentially the same as that in reference 4 , with the only addition being the connection of a metal contact 19 So in this section, we mainly focus on connection to the metal contact. The metal contact has a rectangular cross section in the $(\mathrm{x}, \mathrm{z})$ plane and is infinitely long along the $\mathrm{y}$-axis as shown in Fig. 2. The nanotube lies on the metal contact akin to the experiment of Tans et. all. In reference 2, the nanotube bends over the edge of the metal and the influence of this on transport has recently been modeled by Rochefort et. al.11. In this work, the main focus is to model the coupling between the metal and nanotube. So we assume the nanotube to lie rigidly on the metal and neglect the effect of bending (Fig. 2). A perfectly cylindrical nanotube would touch the metal surface only along a line. To simplify modeling this interface, we stretch the entire circumference of the nanotube over the metal surface and assume coupling between carbon atoms in a sector of the circumference and the metal. Finally, charge self consistency 12 has been neglected.

The transmission and local density of states are calculated in a structure that can be conceptually divided into four parts: section of the nanotube (D), which lies on the metal

electrode (M), semi-infinite regions of the nanotube L and $\mathrm{R}$ [Fig. 2]. The Hamiltonian of 
the system can be written as,

$$
\begin{array}{r}
H=H_{c}+H_{m}+H_{c-m} \text { and } \\
H_{c}=H_{D}+H_{L}+H_{R}+H_{L D}+H_{R D}
\end{array}
$$

where, $H_{c}$ is the pi-electron tight binding Hamiltonian of the nanotube with the on-site potential and hopping parameter between nearest neighbor carbon atoms equal to 0 and $3.1 \mathrm{eV}$ respectively. $H_{L D}$ and $H_{R D}$ are terms in the Hamiltonian coupling D to L and R respectively. $H_{m}$ and $H_{c-m}$ are the free particle and nanotube-metal coupling terms of the Hamiltonian. The Green's function $G^{r}$ is obtained by solving: $\left[E-H_{D}-\Sigma_{L}^{r}-\Sigma_{R}^{r}-\Sigma_{m}^{r}\right] G^{r}(E)=I$, where the self energy $\Sigma_{\alpha}=V_{D \alpha} g_{\alpha}^{r} V_{\alpha D}(\alpha \in \mathrm{L}, \mathrm{R}$ and $\mathrm{M}) \cdot g_{\alpha}^{r}$ is the surface Green's function of lead $\alpha$ and $V_{D \alpha}\left(V_{\alpha D}\right)$ is the coupling between $D(\alpha)$ and $\alpha(D)$. The transmission probability between leads $\alpha$ and $\beta\left[T_{\alpha \beta}\right]$ is given by,

$$
T_{\alpha \beta}(E)=\operatorname{Trace}\left[\Gamma_{\alpha}(E) G^{r}(E) \Gamma_{\beta}(E) G^{a}(E)\right],
$$

where $\Gamma_{\alpha}(E)=2 \pi V_{D \alpha} \rho_{\alpha}(E) V_{\alpha D}$ and $\rho_{\alpha}(E)=-\frac{1}{\pi} \operatorname{Im}\left[g_{\alpha}^{r}(E)\right]$ is the surface density of states of lead $\alpha$.

The Green's function of the metal contact is calculated within the free electron approximation using the procedure outlined below. The metal contact has a rectangular cross section of dimensions $L_{x}$ and $L_{z}$ in the $\mathrm{x}$ and $\mathrm{z}$ directions respectively, and is infinitely long in the $\mathrm{y}$ direction. While the $(\mathrm{y}, \mathrm{z})$-coordinates are assumed to be continuous, the $\mathrm{x}$ coordinate is assumed to be discrete with lattice spacing $a=L_{x} /\left(N_{x}+1\right)$, where $N_{x}$ is the number of lattice points. The wave functions $\left(\Psi_{m k n}\right)$ and eigen values $\left(E_{m k n}\right)$ are given by,

$$
\begin{aligned}
\Psi_{m k n}(r) & =X_{m}(x) Y_{k}(y) Z_{n}(z), \text { where, } \\
X_{m}(x) & =\frac{1}{\sqrt{L_{x}}} \sin \left(\frac{m \pi x}{L_{x}}\right), Y_{k}(y)=\frac{1}{\sqrt{L_{y}}} \exp (i k y), Z_{n}(z)=\frac{1}{\sqrt{L_{z}}} \sin \left(\frac{m \pi z}{L_{z}}\right) \text { and } \\
E_{m k n} & =\frac{\hbar^{2}}{2 m_{o} a^{2}}\left[1-\cos \left(\frac{m \pi}{N_{x}+1}\right)\right]+\frac{\hbar^{2} k^{2}}{2 m_{o}}+\frac{\hbar^{2}}{2 m_{o}}\left(\frac{n \pi}{L_{z}}\right)^{2},
\end{aligned}
$$

where, $m$ and $n$ are positive integers, and $m_{0}$ is the free electron mass. Using Eqns. (6) and (घ) in the following equation for the Green's function, 


$$
g\left(r, r^{\prime}, E\right)=\sum_{m, k, n} \frac{\Psi_{m k n}(r)^{*} \Psi_{m k n}\left(r^{\prime}\right)}{E-E_{m k n}+i \eta}
$$

we obtain,

$$
g\left(r, r^{\prime}, E\right)=-\frac{i m_{o}}{\hbar^{2}} \frac{1}{L_{x} L_{z}} \sum_{m, n} \frac{\exp \left[i k_{I}\left|y-y^{\prime}\right|\right]}{k_{I}} \sin \left(\frac{m \pi x}{L_{x}}\right) \sin \left(\frac{m \pi x^{\prime}}{L_{x}}\right) \sin \left(\frac{n \pi z}{L_{z}}\right) \sin \left(\frac{n \pi z^{\prime}}{L_{z}}\right)
$$

where,

$$
k_{I}=\left\{k^{2}-\left(\frac{n \pi}{L_{z}}\right)^{2}-\frac{1}{a^{2}}\left[1-\cos \left(\frac{m \pi}{N_{x}+1}\right)\right]+i \eta\right\}^{\frac{1}{2}} \text { and } k=\sqrt{\frac{2 m_{o} E}{\hbar^{2}}} .
$$

For carbon nanotubes, the zero of energy $(E=0)$ is taken to lie at the band center. On the other hand, in deriving Eq. (8) the zero of energy corresponded to the band bottom of the free electron metal. In the calculations, there should be only one zero of energy, which we take to lie at the band center of the nanotube. We also neglect charging effects and assume the Fermi energy of the metal to lie at the band center of the nanotube. 13 Then, in the coordinate system where $E=0$ corresponds to the band center of the nanotube, Eq. (8) can be used by transforming,

$$
k=\sqrt{\frac{2 m_{o} E}{\hbar^{2}}} \quad \text { to } \quad k=\sqrt{\frac{2 m_{o} E}{\hbar^{2}}+k_{f}^{2}} \quad \text { in Eq. (9), }
$$

where, $k_{f}$ is the Fermi wave vector of the metal.

The component of the Green's function that enters the calculation of the density of states and transmission probability corresponds to $x=x^{\prime}=a$, the surface of the metal contact on which the nanotube lies. The $(y, z)$ coordinates correspond to the atomic location of the stretched out nanotube lying on the metal. For uniform coupling between the metal and nanotube, we take $V_{D M}=t D_{0}$, where $t$ is the strength of coupling between the free electron metal and a nanotube atom and $D_{0}$ is a diagonal matrix whose dimension is equal to the number of carbon atoms in $D$. The diagonal entry $D_{0}(i, i)=1(0)$ if the carbon atoms make (do not make) contact to the metal. 


\section{RESULTS AND DISCUSSION}

We first present results for dependence of the threshold value of the metal Fermi wave vector on chirality, using armchair and zigzag tubes connected to the metal contact. We then discuss the diameter dependence of conductance using the case of a zigzag tube as an example. Finally, the case of disorder in coupling between the nanotube and metal is considered. We consider only weak coupling between the nanotube and metal. The average value of the non zero diagonal elements of the coupling strength $\Gamma_{M}$ are tabulated in Table 1 for the various values of the metal Fermi wave vector considered. The main guide for the choice of $\Gamma_{M}$ is that it be much smaller than the corresponding coupling strength between two carbon atoms of the nanotube (diagonal component of $\Gamma_{L}$ is approximately equal to $0.3 \mathrm{eV}$ for a $(2,2)$ nanotube). A larger/smaller value of $\Gamma_{M}$ results in a larger/smaller value of transmission in Figures 2 to 5 . We calculate the transmission versus contact length between nanotube and metal for various Fermi wave vectors in the metal and all atoms around the circumference of the tube are assumed to make uniform contact with the metal. We emphasize that when the metal makes contact to only a sector of the nanotube such as in Ref. 2, the results of Fermi wave vector dependence on chirality and the conductance dependence on contact length are still valid. These features depend on the nanotube-metal coupling along the axial direction. So any change due to the finite sector will not qualitatively change the results.

Experiments typically involve transmission of electrons between two metal contacts. The quantity $T_{M L}$ discussed in this section is however the transmission probability between a metal contact and a semi-infinte nanotube (Fig. 2). We consider this quantity because a long nanotube section between two metal contacts requires much more numerically intensive calculations. The physics discussed with regards to $T_{M L}$ in Figs. 3-5 hold in the case of two metallic contacts also, though a direct numerical comparison is not appropriate.

In the case of armchair tubes, when the metal Fermi wave vector $k_{f}$ is smaller than $2 \pi / 3 a_{0}$ $\left(0.85 \AA^{-1}\right), T_{M L}$ does not change significantly with contact length as shown for $k_{f}=0.75 \AA^{-1}$ 
in Fig. 3(a). For values of $k_{f}$ above the threshold, the transmission monotonously increases with an increase in contact length. The monotonic increase is due to weak metal-nanotube coupling, in which case an increase in contact length simply results in an increase in the transition probability to scatter from metal to nanotube.14 The transmission will eventually saturate with increase in contact length as there are only two conducting modes at the band center. For the configuration considered, $T_{M L}$ can have a maximum value of unity. The second feature of Fig. 3(a) is the increase in transmission with increase in $k_{f}$. This can be understood by noting that electrons with a wave vector component along the nanotube axis that is larger than $2 \pi / 3 a_{0}$ scatter from the metal to nanotube, and a larger $k_{f}$ implies a large number of available metal electron states. For the purpose of these calculations, we considered a $(2,2)$ armchair tube; The essential physics would in principle be true for the more realistic $(10,10)$ nanotube also.

The case of zigzag tubes is different because bands at $E=0$ cross at $k=0$. Then, electrons in the metal electrode with any $k_{f}$ (no threshold) can scatter into a metallic zigzag tube. The results for a $(3,0)$ tube are shown in Fig. 3(b). Here, there are two important points. The first point is that as there is no threshold metal Fermi wave vector, the transmission increases monotonically with contact length even for $k_{f}=0.4 \AA^{-1}$, which is smaller than the threshold for armchair tubes. The second point is that the transmission for $k_{f}$ equal to $1.2 \AA^{-1}$ is much smaller than that for armchair tubes [Fig. 3(a); the transmission of the three smaller values of $k_{f}$ have been multiplied by a factor of ten.]. This is because the nanotube wave vector around the circumference $\left(k_{c}\right)$ of a zigzag tube is large; $k_{c}=4 \pi / 3 a_{0}$ for the crossing bands and as a result, the overlap integral [Eq. (11)] is small. As $k_{f}=1.75 \AA^{-1}$ is larger than the threshold for graphite, the transmission probability is larger, and comparable to that for armchair tubes [Fig. 3(b)].

What happens when the diameter increases? In the limit of large diameter, a nanotube is akin to graphene and the threshold $k_{f}$ to couple well with metal should approach $4 \pi / 3 a_{0}$. Numerically, it is difficult to simulate large diameter tubes along with large contact lengths because of time and memory requirements associated with the calculation of $g_{M}^{r}$. To convey 
the main point we consider two simpler cases, the first case compares the transmission probability of the two smallest semi-metallic zigzag tubes with varying contact lengths and the second case considers zigzag tubes of varying diameters with a rather small contact length. Fig. 4 compares the transmission probability versus contact length of the $(3,0)$ and $(6,0)$ nanotubes; The $(6,0)$ nanotube has double the diameter of the $(3,0)$ nanotube. The $(6,0)$ nanotube correspondingly has a smaller transmission and the trend of decrease in transmission will continue with further increase in diameter. The inset is a calculation of transmission probability versus diameter of semi-metallic zigzag tubes for a contact length of $42.6 \stackrel{\circ}{A}$ (ten unit cells). $T_{M L}$ decreases with increase in diameter because wave vector conservation becomes increasingly important with increase in diameter. Shown also in this figure for comparison are $1 /$ diameter and $1 / \sqrt{\text { diameter. }}$

We now address the role of disorder. Disorder in either the nanotube, metal or nanotubemetal coupling will in general result in larger transmission when compared to the disorderfree case. Wave vector conservation is relaxed due to scattering from defects and transmission will increase with increase in contact length even when the metal $k_{f}$ is below the threshold value. We consider the case of disorder in nanotube-metal coupling $\left(H_{c-m}\right)$. Disorder in all elements of the coupling between the nanotube and metal was introduced randomly. The disorder in coupling of atom $i$ to the metal contact can be written as, $t_{i}=\alpha t^{a v}+(1-\alpha) t_{i}^{\text {rand }}$, where $t^{a v}$ is the average value of $t_{i}$ over all sites connected to the metal and $\alpha$ is a fraction between zero and unity. $t_{i}^{\text {rand }}$ is the random component whose average is equal to $t^{a v}$. In Fig. 5 , the two strengths of disorder correspond to $\alpha=0$ and $\alpha=0.5$ (smaller $\alpha$ corresponds to larger disorder), such that $t^{a v}$ has the same value as in Fig. 3(a). For an armchair tube in contact with a metal with $k_{f}=0.75 \AA^{-1}$, the transmission was very small and more importantly did not vary with contact length [Fig. 3(a)]. Introducing disorder changes this trend and causes a monotonic increase in transmission with length of contact [Fig. 5]. Similarly, for large diameter tubes, in the presence of disorder there should be significant transmission when $k_{f}$ is smaller than the threshold $4 \pi / 3 a_{0}$. The requirement of wave vector conservation is also relaxed when the phase coherence length is small. So we expect the 
coupling to improve with decrease in phase coherence length.

\section{CONCLUSIONS}

In this paper, we addressed some aspects of the physics of a nanotube side-contacted to metal, a problem of current importance. Coupling of carbon nanotubes to metal depends on both chirality and diameter. Wave vector conservation of an electron scattered from the nanotube to metal plays a central role in determining the transport properties. The difference between small and large diameter nanotubes is that while in the former wave vector conservation is important only in the axial direction, in the latter it is important in both the axial and circumferential directions. As a result, small diameter armchair and zigzag tubes have a cut-off value of the metal Fermi wave vector equal to $2 \pi / 3 a_{0}$ and zero, respectively. For chiral tubes, the cut-off value of the metal Fermi wave vector lies in between these two limits, with the value decreasing with increase in chiral angle. A large diameter nanotube is akin to a graphene sheet and the cut-off value of the metal Fermi wave vector in this case approaches $4 \pi / 3 a_{0}$ with increase in diameter. Disorder in the metal, nanotube or their coupling relaxes the requirement of k-vector conservation and in general improves coupling. The groups of references 2 and 8 have shown increase in conductance with contact length. In this paper, we discussed two situations that could lead to this. The first situation requires the metal Fermi wave vector to be larger than the threshold discussed in the text and holds even when there is no disorder. The second situation requires disorder in coupling to the metal but there is no restriction on the value of the Fermi wave vector.

\section{ACKNOWLEDGEMENTS}

We acknowledge useful discussion with W. A. de Heer (Georgia Tech), Cees Dekker and Zhen Yao (both of Delft University) and thank J. Tersoff (IBM) for providing us with a preprint of reference 9. We thank Mario Encinosa (FAMU) for many useful comments on 
the manuscript, and Alexei Svizhenko (NASA Ames) for help with commands to parallelize code. 


\section{REFERENCES}

*Corresponding author: anant@nas.nasa.gov.

${ }^{1}$ M. S. Dresselhaus, G. Dresselhaus and P. C. Eklund, Chap. 19 of Science of Fullerenes and Carbon Nanotubes, Academic Press, (1996)

${ }^{2}$ S. J. Tans, M. Devoret, H. Dai, A. Thess, R.E. Smalley, L.J. Geerligs and C. Dekker, Nature 386, 474 (1997).

${ }^{3}$ C. T. White and T. N. Todorov, Nature 393, 240 (1998)

${ }^{4}$ M. P. Anantram and T. R. Govindan, Phys. Rev. B 58, 4882 (1998)

${ }^{5}$ P. J. de Pablo, E. Graugnard, B. Walsh, R. P. Andres, S. Datta and R. Reifenberger, Appl. Phys. Lett. 74, 323 (1999)

${ }^{6}$ H. T. Soh, A. F. Morpurgo, J. Kong, C. M. Marcus, C. F. Quate and H. Dai, Preprint (1999).

${ }^{7}$ D. H. Cobden, M. Bockrath, P. L. McEuen, A. G. Rinzler and R. E. Smalley, Spin Splitting and Even-Odd Effects in Carbon Nanotubes Phys. Rev. Lett 81, 681 (1998)

${ }^{8}$ S. Frank, P. Poncharal, Z. L. Wang and W. A. de Heer, Science 280, 1744 (1998); P. Poncharal, S. Frank, Z. L. Wang and W. A. de Heer, Conductance quantization in multiwalled carbon nanotubes (Preprint)

${ }^{9}$ J. Tersoff, Appl. Phys. Lett. 74, 2122 (1999)

${ }^{10}$ The following references discuss the Green's function formalism used: S. Datta, Electronic Transport in Mesoscopic Systems, Cambridge University Press, Cambridge, U.K (1995); C. Caroli, R. Combescot, P. Nozieres and D. Saint-James, J. Phys. C: Solid St. Phys. 4, 916 (1971); Y. Meir and N.S. Wingreen, Phys. Rev. Lett. 68, 2512 (1992)

${ }^{11}$ A. Rochefort, F. Lesage, D.R. Salahub, and Ph. Avouris, Conductance of Distorted Carbon Nanotubes, cond-mat/9904083 
${ }^{12}$ A. A. Odintsov and Y. Tokura, Contact phenomena in carbon nanotubes, condmat/9906269; K Esfarjani, A. A. Farajian, Y. Hashi and Y. Kawazoe Appl. Phys. Lett. 74, 79 (1999); F. Leonard and J. Tersoff, Novel length scales in nanotube devices, Preprint

${ }^{13}$ One can also take the Fermi energy to lie off band center but this does not play an important role in conveying the main points of this paper.

${ }^{14}$ Note that if the nanotube-metal coupling is strong, then the transmission probability would reach its maximum by contacting just only a few layers along the length. Further increase in contact length will not result in a monotonic increases in transmission with contact length. 


\section{Figure Captions:}

Fig. 1: First Brillouin zone of graphene. Points $P, P^{\prime}, P^{\prime \prime}, Q, Q^{\prime}, Q^{\prime \prime}$ touch the Fermi surface. $a_{0}$ is the lattice vector length of graphene. A metal with Fermi wave vector smaller (inner circle) and larger (outer circle) than $4 \pi / 3 a_{0}$ couples poorly and well to graphene respectively. $\Delta E_{N C}$ is the energy difference between the first non crossing subband below and above $\mathrm{E}=0$.

Fig. 2: A metal making contact to a nanotube. The $(x, z)$ dimensions of the metal form a rectangular cross section with lengths $\left(L_{x}, L_{z}\right)$. The $y$ direction is infinitely long.

Fig. 3: Transmission probability for (a) armchair and (b) zigzag tubes versus contact length. In both cases the largest contact length corresponds to sixty unit cells. The main point of (a) is that for the metal Fermi wave vector smaller than the threshold $2 \pi / 3 a_{0}$, coupling between the nanotube and metal is small and increasing the contact length does not change the transmission probability. For metal Fermi wave vector larger than $2 \pi / 3 a_{0}$, the transmission probability increases with increase in contact length and also with increase in $k_{f}$ for a given contact length. The main point of $(\mathrm{b})$ is that there is no threshold in the metal Fermi wave vector. Even in the case of a small value of the metal Fermi wave vector $\left(0.4 \AA^{-1}\right)$, the transmission increases with increase in the contact length, albeit the magnitude of transmission is small. As in the armchair case, the transmission probability increases with increase in $k_{f}$ for a given contact length. The values of $T_{M L}$ in (b) corresponding to $k_{f}$ equal to $0.4,0.75$ and $1.2 \AA$ are multiplied by a factor of ten.

Fig. 4: Comparison of transmission probability of $(3,0)$ and $(6,0)$ nanotubes versus contact length. The transmission probability decreases with increase in diameter. Inset: The y-axis is $T_{M L}$ for metallic zigzag tubes scaled by $1.0 \mathrm{e}+4$. The solid line is the diameter dependence of $T_{M L}$ for a contact length of $42.6 \stackrel{\circ}{A}$. The upper and lower dashed lines are $1 / \sqrt{\text { diameter }}$ and 1 /diameter dependences, shown for comparison. 
Fig. 5: Comparison of transmission probability versus contact length for a $(2,2)$ armchair tube, with and without disorder in nanotube-metal coupling. The metal Fermi wave vector is $0.75 \AA^{-1}$. Note that for the case without disorder, the transmission is poor and increasing the contact length does not help. Introducing disorder changes this picture and the transmission begins to increase with increase in contact length because k-vector conservation is relaxed. 


\section{TABLES}

Table 1:

\begin{tabular}{|l|l|}
\hline $\begin{array}{c}\text { Metal Fermi } \\
\text { Wave Vector } \\
\left(\AA^{-\mathbf{1}}\right)\end{array}$ & \multicolumn{1}{|c|}{$\Gamma_{\mathbf{M}}(\mathbf{e V})$} \\
\hline \hline 0.4 & $1.1 \times 10^{-4}$ \\
\hline 0.75 & $7.2 \times 10^{-4}$ \\
\hline 0.9 & $1.2 \times 10^{-3}$ \\
\hline 1.2 & $2.9 \times 10^{-3}$ \\
\hline 1.75 & $9.1 \times 10^{-3}$ \\
\hline
\end{tabular}

$\Gamma_{M}$ for the different values of the metal Fermi wave vectors used. 


\section{FIGURES}

Fig. 1 / Anantram

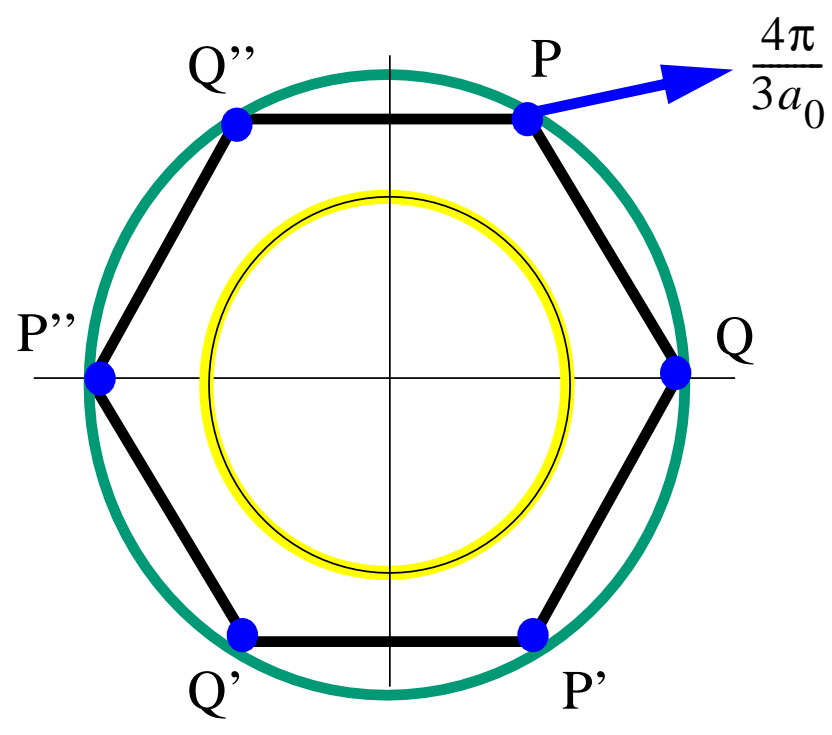


Fig. 2 / Anantram

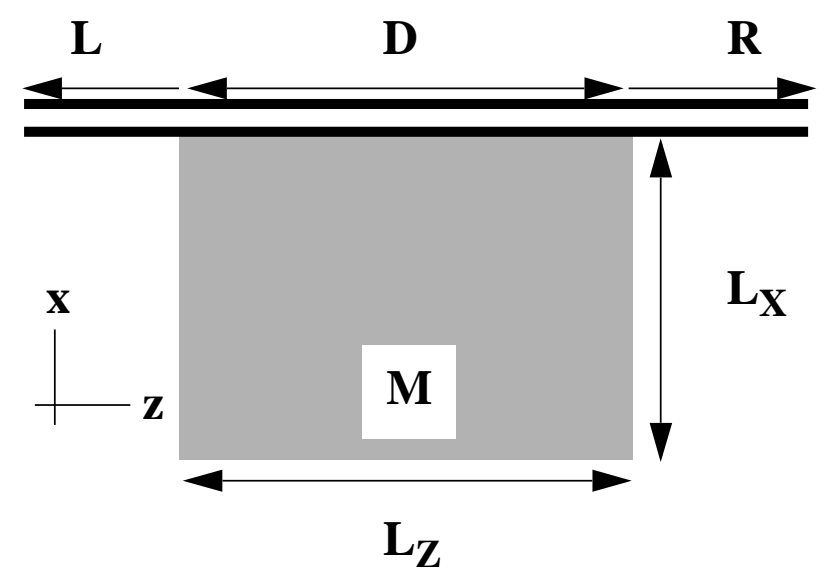


Fig. 3 / Anantram

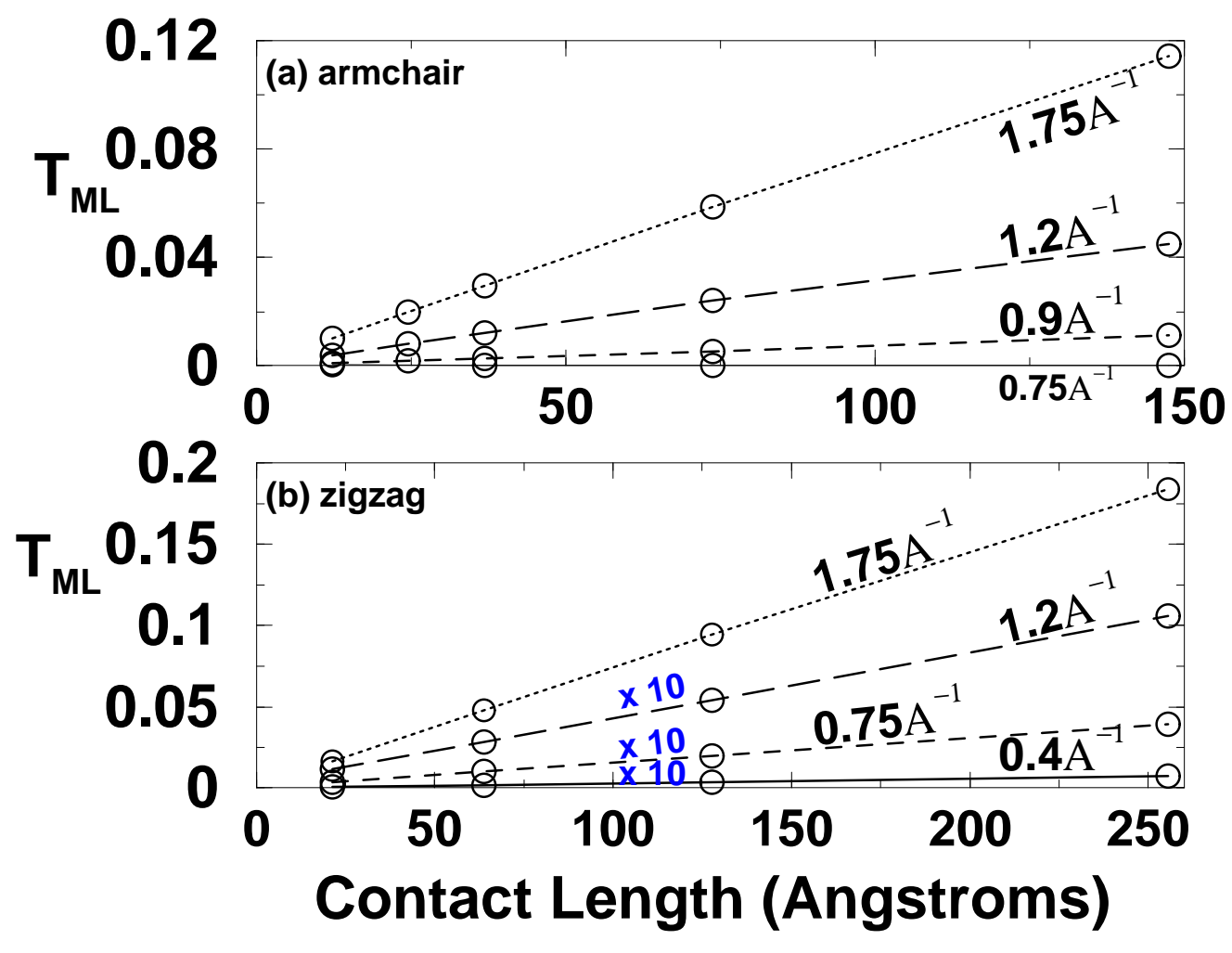


Fig. 4 / Anantram

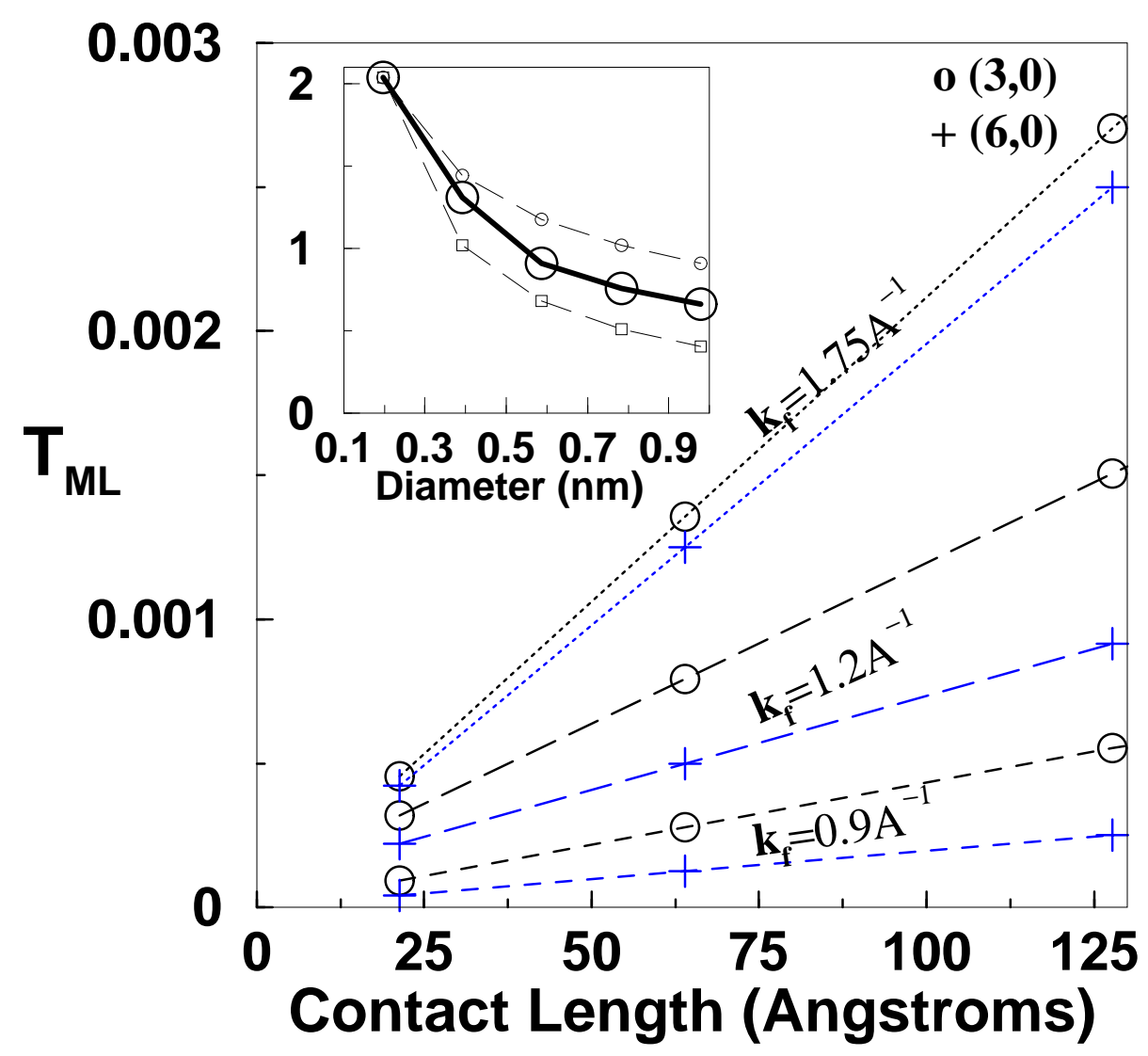


Fig. 5 / Anantram

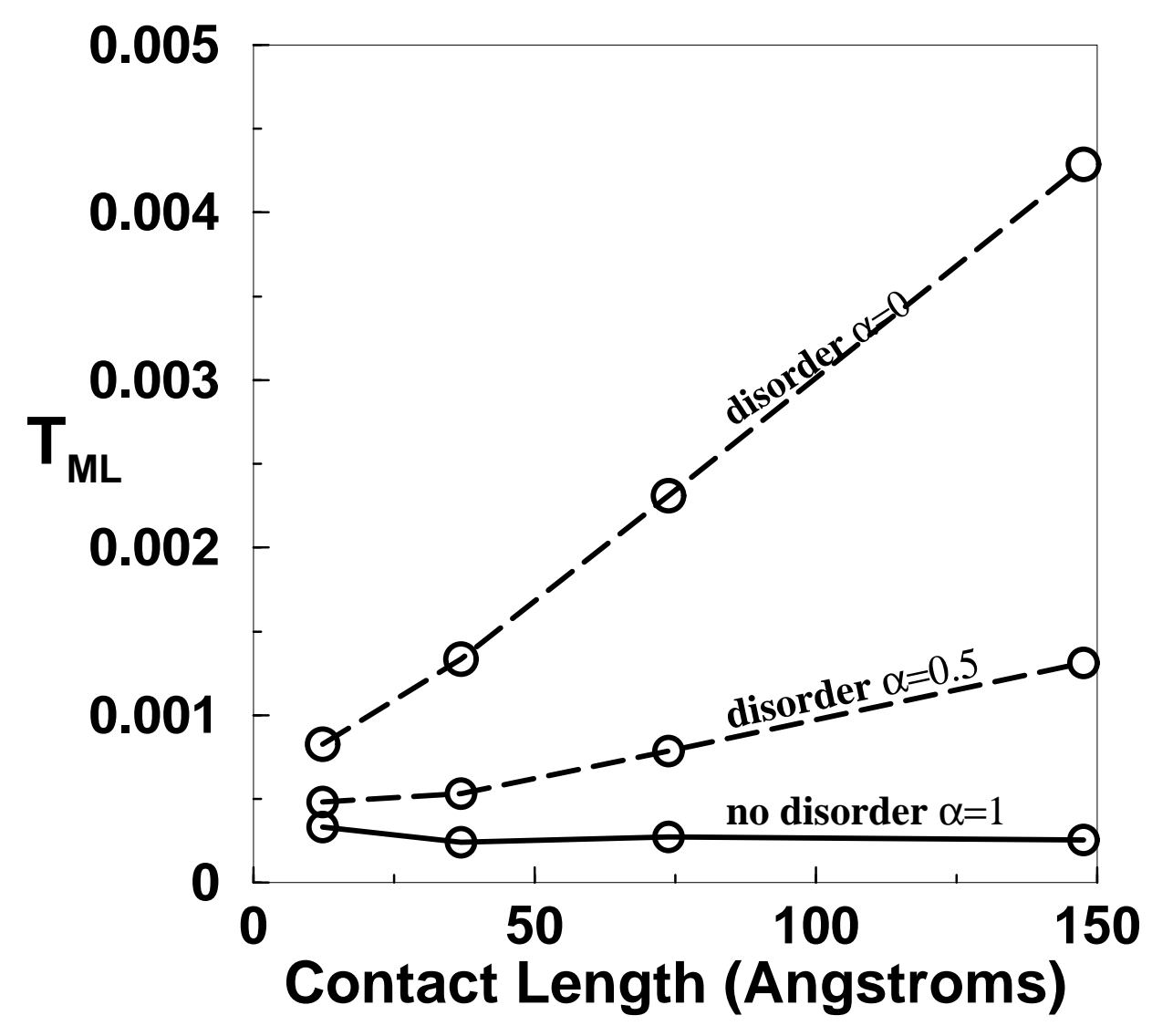

